\title{
Efficacy and Tolerability of Fixed-Combination Brinzolamide/Timolol in Latin American Patients with Open-Angle Glaucoma or Ocular Hypertension Previously on Brimonidine/Timolol Fixed Combination
}

\author{
Arturo Alezzandrini $\cdot$ Douglas Hubatsch $\cdot$ Rene Alfaro
}

To view enhanced content go to www.advancesintherapy.com Received: June 23, 2014 / Published online: August 20, 2014

(C) The Author(s) 2014. This article is published with open access at Springerlink.com

\section{ABSTRACT}

Introduction: Fixed-combination glaucoma medications are commonly used to achieve target intraocular pressure (IOP) reduction in patients uncontrolled with monotherapy; however, ocular discomfort associated with eye drops can decrease adherence. This study assessed the efficacy and tolerability of twicedaily fixed-combination brinzolamide 1\%/ timolol $\quad 0.5 \%$ (BRINZ/TIM-FC) in Latin American patients transitioned from fixedcombination brimonidine $0.2 \% /$ timolol $0.5 \%$ (BRIM/TIM-FC) because of insufficient IOP control or treatment intolerance.

Trial registration: Clinicaltrials.gov \#NCT01518244.

Electronic supplementary material The online version of this article (doi:10.1007/s12325-014-0145-5) contains supplementary material, which is available to authorized users.

A. Alezzandrini $(\bowtie)$

University of Buenos Aires, Buenos Aires, Argentina e-mail: aalezzandrini@oftalmos.com

D. Hubatsch

Alcon Laboratories, Inc., Fort Worth, TX, USA

R. Alfaro

Colego Mexicano de Oftalmologia and Sociedad

Mexicana de Oftalmologia, San Miguel

Chapultepec, Mexico DF, Mexico
Methods: This 8-week, open-label, prospective study was conducted at six sites in Argentina, Chile, and Mexico. Enrolled patients were aged $\geq 18$ years with open-angle glaucoma (including primary, exfoliative, or pigment-dispersion glaucoma) or ocular hypertension with IOP of 19-35 mmHg in $\geq 1$ eye at baseline (on BRIM/ TIM-FC). Patients self-administered BRINZ/TIMFC to both eyes at 8 a.m. and 8 p.m. daily for 8 weeks. The primary and secondary efficacy endpoints were mean IOP change from baseline at week 8 and percentage of patients achieving target IOP $(\leq 18 \mathrm{mmHg})$ at week 8 , respectively. Exploratory endpoints included patient and investigator preference for treatment at week 8. Adverse events (AEs) were assessed as the safety endpoint.

Results: Fifty patients (mean $\pm \mathrm{SD}$ age, $66.7 \pm 11.5$ years) received BRINZ/TIM-FC, and 49 were included in the intent-to-treat population. Mean \pm SD IOP was significantly reduced from baseline after 8 weeks of treatment with BRINZ/TIM-FC $(-3.6 \pm 3.0 \mathrm{mmHg} ; \quad P<0.0001, \quad$ Wilcoxon signed-rank test; $17.1 \%$ reduction). Overall, $55.3 \%$ of patients achieved IOP $\leq 18 \mathrm{mmHg}$ at week 8 . Significantly more patients (89.4\%) and 
investigators (95.7\%) preferred BRINZ/TIM-FC to BRIM/TIM-FC (both $P<0.0001$, exact binomial test). Of the 13 AEs observed, 8 were related to BRINZ/TIM-FC; the most common treatment-related AEs were eye irritation $(n=4)$ and abnormal sensation in the eye $(n=2)$.

Conclusion: BRINZ/TIM-FC provides an effective and well-tolerated treatment option for patients transitioned from BRIM/TIM-FC.

Keywords: $\beta$-Blocker; Carbonic anhydrase inhibitor; Fixed combination; Glaucoma; Intraocular pressure; Latin America; Ocular hypertension; Ophthalmology

\section{INTRODUCTION}

Glaucoma is a leading cause of blindness in Latin America and worldwide [1]. Globally, nearly 80 million people are projected to be diagnosed with glaucoma in 2020, more than 8 million of whom are expected to be from Latin America [1]. Elevated intraocular pressure (IOP) is a major risk factor for primary open-angle glaucoma [2]; the primary treatment approach to slow progression of glaucoma and ocular hypertension is reducing IOP [3]. Several topical IOP-lowering medications exist; these include medications that decrease aqueous humor production and increase aqueous humor outflow (e.g., carbonic anhydrase inhibitors, $\beta$ blockers, $\alpha$-agonists) [3]. Treatment with a single topical agent is often insufficient to control IOP, and many patients require an additional IOP-lowering agent to achieve their targets [3, 4]. Concomitant use of multiple ocular hypotensive agents may be inconvenient and has been associated with increased risk of drug washout [5], decreased adherence and persistence [6, 7], increased exposure to preservatives and risk of preservative-related ocular surface disease $[8$, 9], and potentially higher treatment costs [10].

Fixed-combination medications for glaucoma enable treatment with multiple mechanisms of action in a single topical medication, providing additive IOP-lowering efficacy without increasing treatment complexity or introducing risk of drug washout. The fixed-combination therapy brinzolamide $1 \% /$ timolol $0.5 \%$ (BRINZ/TIMFC; Azarga ${ }^{\circledR}$; Alcon Laboratories, Inc., Fort Worth, TX, USA) combines a carbonic anhydrase inhibitor (CAI) with a $\beta$-blocker and has IOP-lowering efficacy greater than the component monotherapies [11]. Although the safety and efficacy of BRINZ/TIM-FC have been demonstrated in previous studies [12, 13], there are few data on the IOP-lowering efficacy of BRINZ/TIM-FC in patients who transitioned to BRINZ/TIM-FC because of insufficient IOP control or intolerance of a fixed combination containing the $\alpha$-agonist brimonidine. Brimonidine $\quad 0.2 \% /$ timolol $\quad 0.5 \%$ fixedcombination therapy (BRIM/TIM-FC; Combigan ${ }^{\circledR}$; Allergan, Inc., Irvine, CA, USA) has shown efficacy greater than brimonidine or timolol monotherapy alone in patients with glaucoma or ocular hypertension [14].

The objective of this study was to assess the efficacy and tolerability of transitioning to BRINZ/TIM-FC from prior BRIM/TIM-FC in Latin American patients with open-angle glaucoma or ocular hypertension.

\section{METHODS}

\section{Study Design}

This was an 8-week, multicenter, open-label study conducted at six clinical sites in Argentina, Chile, and Mexico from December 
2011 to February 2013 that was designed to evaluate the tolerability and efficacy of changing to BRINZ/TIM-FC from prior BRIM/ TIM-FC in patients with open-angle glaucoma or ocular hypertension. The study consisted of one screening/baseline visit and two follow-up visits conducted after 4 and 8 weeks of treatment. At screening/baseline, patient diagnosis was recorded on a case report form. The form contained a single diagnosis entry for both eyes; in the event that qualifying glaucoma diagnoses differed between eyes, a diagnosis of "non-specified glaucoma" was recorded. Patients received BRINZ/TIM-FC and were instructed to self-administer one drop to each eye twice daily (at 8 a.m. and 8 p.m.) throughout the study. Patients were required to discontinue any prior ocular hypotensive therapy at screening. To represent typical clinical practice patterns and to avoid potentially unsafe increases in IOP, there was no washout period between discontinuation of prior medication and initiation of BRINZ/TIMFC therapy. The following assessments were performed at baseline: best-corrected monocular visual acuity (BCVA) assessment; biomicroscopy of eyelids, conjunctiva, cornea, iris, anterior chamber, and lens; confirmation of open-angle glaucoma by gonioscopy; IOP measurement by Goldmann applanation tonometry; and survey-based assessment of ocular symptoms. BCVA, biomicroscopy, and IOP were also assessed at weeks 4 and 8 . At week 8 , patients completed the ocular symptom survey, global preference response, and adherence questionnaire; investigators completed the global preference response for each patient based on the patient's IOP response to BRINZ/TIM-FC.

The study protocol was approved by all relevant institutional review boards or independent ethics committees, and the study was performed in compliance with the ethical principles of the Declaration of Helsinki and Good Clinical Practice. Before entering the study, all patients provided written informed consent. This trial is registered at Clinicaltrials.gov, number NCT01518244.

\section{Patients}

Eligible patients were aged $\geq 18$ years with an existing diagnosis of ocular hypertension or primary, exfoliative, or pigment-dispersion open-angle glaucoma in $\geq 1$ eye and were on a stable BRIM/TIM-FC treatment regimen for $\geq 30$ days before screening. Other inclusion criteria were IOP between 19 and $35 \mathrm{mmHg}$ in $\geq 1$ eye while on BRIM/TIM-FC, BCVA of $20 / 200$ (Snellen) or better in each eye, and willingness to discontinue use of all other ocular hypotensive agents at screening and throughout the study. Patients must have had IOP considered safe by the investigators in both eyes to ensure clinical stability of vision and the optic nerve. Key exclusion criteria were hypersensitivity to the study medication; presence of a primary or secondary glaucoma other than exfoliative or pigment-dispersion glaucoma; ocular herpes simplex; any abnormality preventing reliable applanation tonometry or examination of the ocular fundus or anterior chamber; corneal dystrophy; conjunctivitis, keratitis, or uveitis; intraocular surgery $\leq 3$ months before screening; cardiopulmonary conditions that precluded safe administration of a topical $\beta$ blocker; use of systemic medications that affect IOP without a stable course for $\geq 7$ days before screening and throughout the study; use of corticosteroids $\leq 30$ days before screening; and risk of visual field or visual acuity worsening as a consequence of participation in the study. 


\section{Efficacy Outcomes}

The primary efficacy variable was change in IOP from baseline to week 8 . The percentage of patients with IOP $\leq 18 \mathrm{mmHg}$ at week 8 was the secondary efficacy variable. IOP was assessed by the same operator with the same tonometer at least $2 \mathrm{~h}$ after the morning instillation of the study drug and at approximately the same time of day for all visits. Exploratory efficacy variables were ocular symptom survey responses by visit and change from baseline to week 8 , adherence questionnaire responses at week 8 , and patient and investigator global preference responses at week 8. The global preference questionnaire consisted of a single question with a forced-choice binary response (i.e., preferred study medication [BRINZ/TIMFC] or preferred prior medication [BRIM/TIMFC]).

\section{Safety Outcomes}

Safety was assessed by monitoring adverse event (AE) reports. Ocular signs (evaluated by slitlamp biomicroscopy) and BCVA were evaluated for each eye at all study visits.

\section{Statistical Analysis}

If only one eye was eligible for inclusion and treatment, this eye was selected for the efficacy analysis; if both eyes were eligible, the worse eye (i.e., the eye with the higher IOP at baseline) was selected. If both eyes had equal IOP at baseline, the right eye was selected. Efficacy outcomes were analyzed in the intent-to-treat (ITT) population (i.e., patients who received BRINZ/TIM-FC and had $\geq 1$ on-therapy study visit) and the per-protocol population (i.e., all subjects who received BRINZ/TIM-FC, completed all study visits, and satisfied all inclusion/exclusion criteria); missing data were not imputed. For the primary efficacy variable, differences in IOP measurements between baseline and week 8 were compared using Wilcoxon signed-rank tests. To assess the effect of time on IOP, changes in IOP across visits were evaluated using a repeated-measures analysis of variance. Ordinal responses from the ocular symptom survey were converted to a numeric scale $(0=$ none to $4=$ severe $)$; differences from baseline to week 8 were assessed using Wilcoxon signed-rank tests and sign tests. Patient and investigator responses to the global preference questionnaire were assessed with exact binomial tests. Safety analyses were performed in the safety population (i.e., all patients who received $\geq 1$ dose of BRINZ/TIM-FC). Descriptive statistics were provided for demographic, adherence, and safety endpoints. Statistical analyses were performed using $\mathrm{SAS}^{\circledR}$, version 9.2 (SAS Institute Inc., Cary, NC, USA); all statistical tests were two-tailed with a significance level of 0.05 .

\section{RESULTS}

\section{Patients}

Fifty patients were screened and enrolled in the study and included in the safety population. Open anterior chamber angle was confirmed for all patients. Mean \pm SD patient age was $66.7 \pm 11.5$ years (range $29-87$ years); $61.2 \%$ of patients were female, and $89.8 \%$ were Hispanic (Table 1). Three patients discontinued because of AEs (keratitis, bacterial conjunctivitis, and eye irritation); one patient discontinued between baseline and week 4 and was excluded from the ITT analysis $(n=49)$, and 
Table 1 Patient demographics (intent-to-treat population)

\begin{tabular}{|c|c|}
\hline Characteristic & BRINZ/TIM-FC $(n=49)$ \\
\hline \multicolumn{2}{|l|}{ Age, years } \\
\hline Mean $\pm S D$ & $66.7 \pm 11.5$ \\
\hline Range & $29-87$ \\
\hline \multicolumn{2}{|l|}{ Sex, $n(\%)$} \\
\hline Female & $30(61.2)$ \\
\hline Male & $19(38.8)$ \\
\hline \multicolumn{2}{|l|}{ Race, $n(\%)$} \\
\hline Hispanic & $44(89.8)$ \\
\hline Caucasian & $5(10.2)$ \\
\hline Weight, mean $\pm \mathrm{SD}$, kg & $71.7 \pm 15.3$ \\
\hline Height, mean $\pm \mathrm{SD}, \mathrm{cm}$ & $163.9 \pm 11.1$ \\
\hline $\mathrm{BMI}$, mean $\pm \mathrm{SD}, \mathrm{kg} / \mathrm{m}^{2}$ & $26.6 \pm 4.7$ \\
\hline \multicolumn{2}{|l|}{ Diagnosis, $n(\%)^{a}$} \\
\hline Open-angle glaucoma & $26(53.1)$ \\
\hline Non-specified glaucoma ${ }^{b}$ & $22(44.9)$ \\
\hline Ocular hypertension & $4(8.2)$ \\
\hline \multicolumn{2}{|c|}{$\begin{array}{l}\text { BMI Body mass index, BRINZ/TIM-FC fixed- } \\
\text { combination brinzolamide } 1 \% / \text { timolol } 0.5 \% \\
\text { a Two diagnoses were recorded for some patients, causing } \\
\text { the total number of diagnoses to exceed } 49 \\
\text { b Recorded for each patient who had a single glaucoma } \\
\text { entry for both eyes; type of glaucoma was not allowed to be } \\
\text { specified for each eye. Two patients presented with } \\
\text { pseudoexfoliation glaucoma and were included as non- } \\
\text { specified glaucoma; one patient had open-angle glaucoma } \\
\text { in both eyes but was assigned to the non-specified } \\
\text { glaucoma group because of a coding error }\end{array}$} \\
\hline
\end{tabular}

two patients discontinued between weeks 4 and 8.

\section{Efficacy}

Efficacy data were similar in the ITT and perprotocol populations; results for the ITT population are presented. At baseline (on $\mathrm{BRIM} / \mathrm{TIM}-\mathrm{FC} ; n=49$ ), mean $\pm \mathrm{SD}$ IOP was $21.2 \pm 2.5 \mathrm{mmHg}$. Mean $\pm \mathrm{SD}$ absolute IOP reduction from baseline to week 8 (on BRINZ/ TIM-FC; $\quad n=47) \quad$ was $\quad-3.6 \pm 3.0 \mathrm{mmHg}$ $(P<0.0001)$; mean \pm SD percent IOP change from baseline to week 8 was $-17.1 \pm 13.7 \%$. Mean IOP decreased from baseline to week 4 $(n=49)$ and was maintained through week 8 (Fig. 1). At week 8, 55.3\% of patients ( $n=26 / 47)$ achieved the target IOP of $\leq 18 \mathrm{mmHg}$.

The proportion of patients reporting the symptoms described in the ocular symptom survey decreased from baseline to week 8 , except for crusting around the eyes, which increased from $12.2 \%(n=6 / 49)$ to $27.7 \%$ $(n=13 / 47 ; \quad$ Table 2$)$. Dry eyes; excessive tearing; and itching of the eyes, eyelids, or the area around the eyes were significantly improved from baseline to week 8 (all $P<0.05$; Table 2).

Most patients $(89.4 \%, n=42 / 47)$ preferred BRINZ/TIM-FC to BRIM/TIM-FC $(P<0.0001)$. Investigators preferred BRINZ/TIM-FC to BRIM/TIM-FC for nearly all patients $(95.7 \%$, $n=45 / 47 ; P<0.0001)$.

All but 1 patient $(97.9 \%, n=46 / 47)$ were very confident that they would adhere to their preferred glaucoma medication if it was prescribed by their doctor, compared with $61.7 \%(n=29 / 47)$ who were very confident that they would adhere to their non-preferred medication if it was prescribed (Table 3 ). In total, $87.2 \%(n=41 / 47)$ of patients were very confident they would use a prescribed medication if it did not make their eyes burn or sting, whereas $59.6 \%(n=28 / 47)$ of patients were very confident that they would use a prescribed medication if it did cause such symptoms (Table 3). 


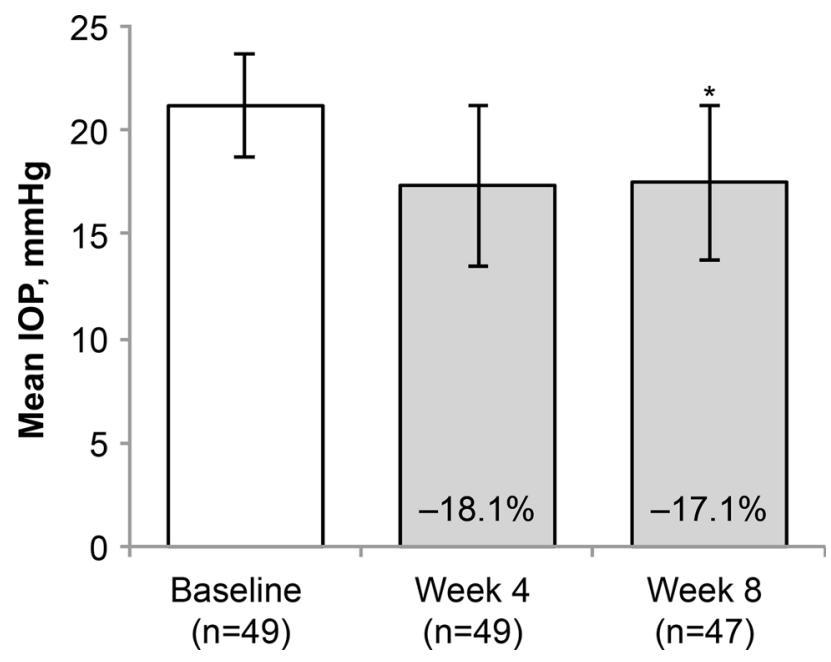

Fig. 1 Mean \pm SD IOP throughout the study (intent-totreat population). Percent IOP reduction from baseline to week 4 and week 8 is indicated inside bars; gray bars indicate IOP with BRINZ/TIM-FC treatment.
${ }^{*} P<0.0001$, week 8 versus baseline; Wilcoxon test. Overall effect of time on IOP, $P<0.0001$; repeated-measures analysis of variance. BRINZ/TIM-FC fixed-combination brinzolamide $1 \% /$ timolol $0.5 \%$, IOP intraocular pressure

\section{Safety}

A total of 13 AEs were reported for 10 patients (Table 4). Most AEs were mild or moderate in intensity, and all resolved with or without treatment. Eight AEs in five patients were considered related to BRINZ/TIM-FC; the most common treatment-related AEs were eye irritation $(n=4)$ and abnormal sensation in the eye $(n=2)$. There were no serious AEs.

Approximately $50 \%$ of patients showed lens abnormalities in the slit-lamp examination in both eyes at baseline and follow-up visits; the most common lens abnormality was mild nuclear opacity. Findings for cornea, iris/ anterior chamber, eyelids, and conjunctiva were generally unchanged from baseline at weeks 4 and 8. No marked differences in BCVA from baseline to week 4 or week 8 were observed.

\section{DISCUSSION}

Fixed-combination therapies for glaucoma provide an effective and convenient therapeutic option for patients who cannot maintain target IOP reduction on monotherapy alone. BRINZ/TIM-FC and BRIM/ TIM-FC each provide two ocular hypotensive agents in a single eye drop and achieve greater IOP reductions than their individual components alone. Compared with concomitant therapy with their unfixed components, fixed-combination therapies provide similar IOP-lowering efficacy, decreased treatment complexity, lower risk of drug washout, decreased preservative exposure and preservative-related ocular discomfort, and may reduce treatment costs $[5,8-10]$. By simplifying treatment regimens and decreasing risk of preservative-related ocular side effects, 
Table 2 Ocular symptom survey by visit and change from baseline (intent-to-treat population)

\begin{tabular}{|c|c|c|c|c|}
\hline \multirow[t]{2}{*}{ Question } & \multicolumn{2}{|c|}{ Patients, $n(\%)$} & \multicolumn{2}{|c|}{$\begin{array}{l}\text { Change from baseline } \\
\text { to week } 8^{b}(n=47)\end{array}$} \\
\hline & $\begin{array}{l}\text { Baseline }^{\mathrm{a}} \\
(n=49)\end{array}$ & $\begin{array}{l}\text { Week } 8^{\mathbf{a}} \\
(n=47)\end{array}$ & $\overline{\text { Mean } \pm \text { SD }}$ & $P$ value \\
\hline Have you had dry eyes since your last visit? & $17(35.4)^{\mathrm{c}}$ & $9(19.1)$ & $-0.35 \pm 1.16$ & $<0.0486^{\mathrm{d}}$ \\
\hline $\begin{array}{l}\text { Have you had pain in or around your eyes when in the light } \\
\text { since your last visit? }\end{array}$ & $16(32.7)$ & $11(23.4)$ & $-0.30 \pm 1.25$ & $<0.1321^{\mathrm{d}}$ \\
\hline Have you teared more than normal since your last visit? & $16(32.7)$ & $4(8.5)$ & $-0.34 \pm 0.94$ & $0.0097^{\mathrm{d}}$ \\
\hline Did your eye drops sting or burn when you instilled them? & $26(53.1)$ & $18(38.3)$ & $-0.53 \pm 1.54$ & $0.2295^{\mathrm{e}}$ \\
\hline Have you had crusting around your eyes since your last visit? & $6(12.2)$ & $13(27.7)$ & $0.30 \pm 1.28$ & $0.1455^{\mathrm{d}}$ \\
\hline $\begin{array}{l}\text { Have you had itching of eyes, eyelids, or the area around } \\
\text { your eyes since your last visit? }\end{array}$ & $19(38.8)$ & $8(17.0)$ & $-0.55 \pm 0.95$ & $0.0007^{\mathrm{e}}$ \\
\hline $\begin{array}{l}\text { Have you had a feeling of irritation in your eyes since your } \\
\text { last visit? }\end{array}$ & $18(36.7)$ & $8(17.0)$ & $-0.37 \pm 1.18$ & $0.0509^{\mathrm{d}}$ \\
\hline Have you noticed redness in your treated eyes? & $11(22.4)$ & $10(21.3)$ & $-0.23 \pm 1.34$ & $0.2370^{\mathrm{d}}$ \\
\hline $\begin{array}{l}\text { Have other people commented about redness in your } \\
\text { treated eyes? }\end{array}$ & $15(30.6)$ & $8(17.0)$ & NA & NA \\
\hline \multicolumn{5}{|l|}{ How easy is it for you to instill your eye drops in your eyes? } \\
\hline Very difficult & $2(4.1)$ & $0(0.0)$ & NA & NA \\
\hline Difficult & $2(4.1)$ & $4(8.5)$ & NA & NA \\
\hline Easy & $19(38.8)$ & $18(38.3)$ & NA & NA \\
\hline Very easy & $26(53.1)$ & $25(53.2)$ & NA & NA \\
\hline
\end{tabular}

$N A$ Not applicable

${ }^{a}$ Values represent "yes" responses to each question, with the exception of the question "How easy is it for you to instill your eye drops in your eyes?” for which responses regarding levels of ease with instillation are shown

b Values represent change from baseline to week 8 in answers on a 5 -point scale of symptom severity $(0=$ none; $1=$ minimal; $2=$ mild; $3=$ moderate; $4=$ severe). Negative values indicate an improvement in ocular symptoms

c $n=48$

d Wilcoxon test

e Sign test

fixed combinations may also promote improved treatment adherence.

In the current study of Latin American patients with open-angle glaucoma or ocular hypertension, a significant reduction in IOP was observed after patients were transitioned from twice-daily BRIM/TIM-FC to twice-daily BRINZ/ TIM-FC; mean IOP decreased from baseline to week 8 by $3.6 \mathrm{mmHg}$. All enrolled patients had a minimum IOP $\geq 19 \mathrm{mmHg}$ with BRIM/TIMFC, and more than half of patients $(n=26 / 47)$ achieved the target IOP of $\leq 18 \mathrm{mmHg}$ after 8 weeks of treatment with BRINZ/TIM-FC. Results of the ocular symptom survey showed that patients reported greater ocular comfort with BRINZ/TIM-FC compared with BRIM/TIM- 
Table 3 Adherence questionnaire (intent-to-treat population)

\begin{tabular}{lc}
\hline Question, $n(\%)$ & Week $8(n=47)$ \\
\hline How confident is the patient that he/she will use glaucoma \\
medication as prescribed if the doctor: \\
Prescribed the medication preferred in the study \\
Not at all confident & 0 \\
Somewhat confident & $1(2.1)$ \\
Very confident & $46(97.9)$ \\
Prescribed the medication not preferred in the study \\
Not at all confident & $12(25.5)$ \\
Somewhat confident & $6(12.8)$ \\
Very confident & $29(61.7)$ \\
Prescribed a medication that makes the eyes burn or sting \\
Not at all confident & $11(23.4)$ \\
Somewhat confident & $8(17.0)$ \\
Very confident & $28(59.6)$ \\
Prescribed a medication that does not make the eyes burn \\
or sting
\end{tabular}

FC. Nearly $90 \%$ of patients preferred BRINZ/ TIM-FC to BRIM/TIM-FC, possibly because of the improved tolerability and comfort reported for BRINZ/TIM-FC; investigators preferred BRINZ/TIM-FC for nearly all patients (95.7\%). No substantial changes in ocular signs or BCVA were observed throughout the study, and the safety profile of BRINZ/TIM-FC was similar to previous reports $[12,13]$. All AEs were consistent with AEs previously reported with topical $\beta$-blockers and CAIs.

The observed reduction in IOP with BRINZ/ TIM-FC was evident after 4 weeks of treatment and was maintained through 8 weeks. Mean IOP with BRINZ/TIM-FC treatment was
$17.35 \mathrm{mmHg}$ and $17.51 \mathrm{mmHg}$ at weeks 4 and 8 , respectively; at week 8 , mean IOP was reduced by $17.1 \%$ from baseline levels $(21.2 \mathrm{mmHg})$, when patients were receiving BRIM/TIM-FC. Furthermore, whereas no patients had IOP $<19 \mathrm{mmHg}$ at baseline, $55.3 \%$ of patients achieved the target IOP of $\leq 18 \mathrm{mmHg}$ after 8 weeks of BRINZ/TIM-FC treatment. Maintaining IOP $\leq 18 \mathrm{mmHg}$ has been associated with less disease progression and increased stability of visual fields over several years of patient follow-up. A metaanalysis of five studies of patients with openangle glaucoma found that elevated IOP was a significant risk factor for disease progression over 5 years; visual fields were stable in $82 \%$ of patients with mean IOP of $13-17 \mathrm{mmHg}$ compared with only $49 \%$ of patients with mean IOP $>18 \mathrm{mmHg}$ [15]. Mean IOP was $20.0 \mathrm{mmHg}$ in patients with signs of disease progression compared with $17.1 \mathrm{mmHg}$ in patients without progression [15]. Likewise, the Advanced Glaucoma Intervention Study comparison of surgical interventions for glaucoma demonstrated that visual field progression was decreased in patients who consistently maintained IOP levels $\leq 18 \mathrm{mmHg}$ over 6 years compared with patients who had higher IOP levels [16]. In the Canadian Glaucoma Study, among 249 patients with open-angle glaucoma, those with mean IOP $>17 \mathrm{mmHg}$ had significantly higher cumulative progression compared with those with mean IOP $<15 \mathrm{mmHg}$ [17]; this study also found that the risk of progression increased by $19 \%$ for every $1-\mathrm{mmHg}$ increase in mean IOP [17]. Similarly, the Early Manifest Glaucoma Trial demonstrated that the risk of progression decreased by $\sim 10 \%$ for every $1-\mathrm{mmHg}$ decrease in IOP from baseline at follow-up visits [18]. This finding was further supported by the Canadian Glaucoma Study follow-up 
Table 4 Summary of adverse events (safety population)

\begin{tabular}{ll}
\hline Adverse events, $\boldsymbol{n}$ (\%) & $\begin{array}{l}\text { BRINZ/TIM- } \\
\text { FC } \\
(\boldsymbol{n}=\mathbf{5 0})\end{array}$ \\
\hline Patients with $\geq 1 \mathrm{AE}$ & $10(20.0)$ \\
Treatment-emergent AEs: & \\
Eye irritation & $4(8.0)$ \\
Abnormal sensation in eye & $2(4.0)$ \\
Conjunctivitis & $2(4.0)$ \\
Foreign body sensation in eyes & $1(2.0)$ \\
Keratitis & $1(2.0)$ \\
Bacterial conjunctivitis & $1(2.0)$ \\
Viral conjunctivitis & $1(2.0)$ \\
Gastroenteritis & $1(2.0)$ \\
Patients with $\geq 1$ treatment-related & $5(10.0)$ \\
AE & \\
Treatment-related AEs: & \\
Eye irritation & $4(8.0)$ \\
Abnormal sensation & $2(4.0)$ \\
Foreign body sensation & $1(2.0)$ \\
Keratitis & $1(2.0)$ \\
\hline
\end{tabular}

$A E$ Adverse event, BRINZ/TIM-FC fixed-combination brinzolamide $1 \% /$ timolol $0.5 \%$

report, which showed that even a modest IOP reduction in patients with glaucoma progression significantly slowed the rate of visual field decline [19]. In the current study, BRINZ/TIM-FC therapy led to significant IOP reductions from levels maintained with BRIM/ TIM-FC. This finding is consistent with the results of a systematic review and meta-analysis that demonstrated greater mean diurnal IOP reduction with BRINZ/TIM-FC compared with BRIM/TIM-FC (-8.3 mmHg versus $-6.6 \mathrm{mmHg}$, respectively; overall difference, $1.7 \mathrm{mmHg}$ ) [20]. The difference in IOP with BRINZ/TIM-FC versus BRIM/TIM-FC in the current study was of greater magnitude than was previously reported [20]; however, the current study did not control for potential non-compliance with prior BRIM/TIM-FC.

The safety and tolerability profiles of BRINZ/ TIM-FC and BRIM/TIM-FC in the current study were similar to previous reports. In a large, multicenter, observational study of more than 14,000 patients throughout Germany who transitioned to BRINZ/TIM-FC from prior IOPlowering medications, nearly $90 \%$ of patients judged the tolerability of BRINZ/TIM-FC positively [13]. A total of $75.9 \%$ of evaluable patients reported a preference for BRINZ/TIMFC compared with $8.6 \%$ who reported a preference for their previous therapy [13]. Of more than 200 patients who were transitioned from BRIM/TIM-FC to BRINZ/TIM-FC, more patients rated BRINZ/TIM-FC tolerability favorably $(86.5 \%)$ compared with BRIM/TIMFC tolerability (32.1\%); similar to the results of the current study, more patients reported a preference for BRINZ/TIM-FC (75.8\%) compared with BRIM/TIM-FC (6.6\%) [13].

The open-label, single-arm design, lack of active control, 8-week follow-up, and subjective nature of the surveys are potential limitations of this study. IOP was assessed and recorded by the same operator for each patient throughout the study, which could have introduced bias based on the unmasked nature of the study. The single baseline IOP assessment for determination of patient eligibility did not allow adjustment for potential noncompliance with BRIM/TIM-FC at screening, and regression to the mean may have affected study results. Inclusion of patients with IOP considered safe for study participation may have introduced patient selection bias. Adherence with BRINZ/TIM-FC may have been higher because of the effect of participation in a clinical trial. Furthermore, the preference questionnaire consisted of a binary choice question; therefore, 
patients who may have had no medication preference were prompted to choose between BRIM/TIM-FC and BRINZ/TIM-FC. Prior intolerance to BRIM/TIM-FC may have introduced bias to patients' questionnaire responses. Future studies including a crossover design and longer follow-up durations would provide valuable data regarding long-term treatment adherence, tolerability, efficacy, and medication preference in this patient population.

\section{CONCLUSION}

In this study of patients transitioning from BRIM/TIM-FC to BRINZ/TIM-FC because of insufficient IOP reduction or intolerance, BRINZ/TIM-FC significantly reduced IOP after 4 weeks of treatment and maintained IOP reductions through 8 weeks; more than half of the patients studied achieved target IOP (i.e., $\leq 18 \mathrm{mmHg}$ ) after 8 weeks of treatment. BRINZ/ TIM-FC was preferred by significantly more patients and investigators and was associated with improved tolerability compared with BRIM/TIM-FC. These results suggest that BRINZ/TIM-FC is an effective and welltolerated option for patients transitioning from BRIM/TIM-FC.

\section{ACKNOWLEDGMENTS}

This study and article processing charges were sponsored by Alcon Research, Ltd., Fort Worth, TX, USA. Medical writing support was provided by Jane A. Phillips, PhD, and Heather D. Starkey, $\mathrm{PhD}$, of Complete Healthcare Communications, Inc. (Chadds Ford, PA, USA) and was funded by Alcon. All named authors meet the ICMJE criteria for authorship for this manuscript, take responsibility for the integrity of the work as a whole, and have given final approval for the version to be published. All authors had full access to all of the data in this study and take complete responsibility for the integrity of the data and accuracy of the data analysis.

Conflict of interest. Arturo Alezzandrini and Rene Alfaro have no conflicts to disclose. Douglas Hubatsch is an employee of Alcon.

Compliance with ethics guidelines. All procedures followed were in accordance with the ethical standards of the responsible committee on human experimentation (institutional and national) and with the Helsinki Declaration of 1975 , as revised in 2000 and 2008. Informed consent was obtained from all patients for being included in the study.

Funding. This study was sponsored by Alcon Research, Ltd., Fort Worth, TX, USA.

Open Access. This article is distributed under the terms of the Creative Commons Attribution Noncommercial License which permits any noncommercial use, distribution, and reproduction in any medium, provided the original author(s) and the source are credited.

\section{REFERENCES}

1. Quigley HA, Broman AT. The number of people with glaucoma worldwide in 2010 and 2020. Br J Ophthalmol. 2006;90:262-7.

2. Sommer A, Tielsch JM, Katz J, et al. Relationship between intraocular pressure and primary open angle glaucoma among white and black Americans. The Baltimore Eye Survey. Arch Ophthalmol. 1991;109:1090-5.

3. Webers CA, Beckers HJ, Nuijts RM, Schouten JS. Pharmacological management of primary openangle glaucoma: second-line options and beyond. Drugs Aging. 2008;25:729-59. 
4. Kass MA, Heuer DK, Higginbotham EJ, et al. The Ocular Hypertension Treatment Study: a randomized trial determines that topical ocular hypotensive medication delays or prevents the onset of primary open-angle glaucoma. Arch Ophthalmol. 2002;120:701-13.

5. Chrai SS, Makoid MC, Eriksen SP, Robinson JR. Drop size and initial dosing frequency problems of topically applied ophthalmic drugs. J Pharm Sci. 1974;63:333-8.

6. Djafari F, Lesk MR, Harasymowycz PJ, Desjardins D, Lachaine J. Determinants of adherence to glaucoma medical therapy in a long-term patient population. J Glaucoma. 2009;18:238-43.

7. Reardon G, Kotak S, Schwartz GF. Objective assessment of compliance and persistence among patients treated for glaucoma and ocular hypertension: a systematic review. Patient Prefer Adherence. 2011;5:441-63.

8. Skalicky SE, Goldberg I, McCluskey P. Ocular surface disease and quality of life in patients with glaucoma. Am J Ophthalmol. 2012;153(1-9):e2.

9. Leung EW, Medeiros FA, Weinreb RN. Prevalence of ocular surface disease in glaucoma patients. J Glaucoma. 2008;17:350-5.

10. Hommer A. Role of fixed combinations in the management of open-angle glaucoma. Expert Rev Pharmacoecon Outcomes Res. 2011;11:91-9.

11. Kaback M, Scoper SV, Arzeno G, et al. Intraocular pressure-lowering efficacy of brinzolamide $1 \% /$ timolol $0.5 \%$ fixed combination compared with brinzolamide $1 \%$ and timolol $0.5 \%$. Ophthalmology. 2008;115:1728-34.

12. Manni G, Denis $P$, Chew $P$, et al. The safety and efficacy of brinzolamide $1 \%$ /timolol $0.5 \%$ fixed combination versus dorzolamide $2 \%$ /timolol $0.5 \%$ in patients with open-angle glaucoma or ocular hypertension. J Glaucoma. 2009;18:293-300.
13. Lanzl I, Raber T. Efficacy and tolerability of the fixed combination of brinzolamide $1 \%$ and timolol $0.5 \%$ in daily practice. Clin Ophthalmol. 2011;5:291-8.

14. Craven ER, Walters TR, Williams $\mathrm{R}$, et al. Brimonidine and timolol fixed-combination therapy versus monotherapy: a 3-month randomized trial in patients with glaucoma or ocular hypertension. J Ocul Pharmacol Ther. 2005;21:337-48.

15. Stewart WC, Kolker AE, Sharpe ED, et al. Long-term progression at individual mean intraocular pressure levels in primary open-angle and exfoliative glaucoma. Eur J Ophthalmol. 2008;18:765-70.

16. The Advanced Glaucoma Intervention Study (AGIS) Investigators. The Advanced Glaucoma Intervention Study (AGIS): 7. The relationship between control of intraocular pressure and visual field deterioration. Am J Ophthalmol. 2000; 130:429-40.

17. Chauhan BC, Mikelberg FS, Balaszi AG, et al. Canadian Glaucoma Study: 2. Risk factors for the progression of open-angle glaucoma. Arch Ophthalmol. 2008;126:1030-6.

18. Leske MC, Heijl A, Hussein M, et al. Factors for glaucoma progression and the effect of treatment: the Early Manifest Glaucoma Trial. Arch Ophthalmol. 2003;121:48-56.

19. Chauhan BC, Mikelberg FS, Artes PH, et al. Canadian Glaucoma Study: 3. Impact of risk factors and intraocular pressure reduction on the rates of visual field change. Arch Ophthalmol. 2010;128:1249-55.

20. Cheng JW, Cheng SW, Gao LD, Lu GC, Wei RL. Intraocular pressure-lowering effects of commonly used fixed-combination drugs with timolol: a systematic review and meta-analysis. PLoS One. 2012;7:e45079. 